Bundesweite Umfrage zur CT-Expositionspraxis

\title{
Deutsche Röntgengesellschaft, Berufsverband der Deutschen Radiologen und Bundesamt für Strahlenschutz bitten um Informationen
}

Die Deutsche Röntgengesellschaft (DRG) und der Berufsverband der Deutschen Radiologen (BDR) führen gemeinsam mit dem Bundesamt für Strahlenschutz (BfS) eine bundesweite Umfrage zur aktuellen CT-Expositionspraxis in Deutschland durch. Die Einreichungsfrist für diese Umfrage wurde bis zum 31. Oktober 2012 verlängert.

Ziel der Erhebung ist es, die anhaltenden Diskussionen über die Strahlenexposition aus CT-Untersuchungen zu versachlichen. Alle Ärzte, Medizinphysiker und MTRAs die an CT-Geräten in Kliniken und Praxen arbeiten, sind eingeladen, an dieser bundesweiten Erhebung teilzunehmen und den Fragebogen „CT-Untersuchungen an Erwachsenen“ bzw. „CT-Untersuchungen an Kindern" auszufüllen. Insbesondere sollen dosisrelevante Untersuchungsparameter für ein breites Spektrum an Standarduntersuchungen an Erwachsenen und Kindern bei möglichst allen deutschen CT-Betreibern erfasst und ausgewertet werden. Weitere Informationen finden Sie auf der
Homepage der Deutschen Röntgengesellschaft unter: www.drg.de/component/ content/article/8/635

Dort stehen auch die Fragebögen, um deren Ausfüllung wir Sie bitten, zum Download bereit. Ihre Antwort richten Sie bitte bis zum 31. Oktober 2012 an folgende E-Mailadresse: dosisct@drg.de

Selbstverständlich werden Ihre Angaben streng vertraulich behandelt. Nach erfolgter Auswertung der Umfragedaten werden wir Sie informieren, wie die Dosiswerte in Ihrer Einrichtung im Vergleich zur Gesamtgruppe einzuordnen sind.

Für Ihre Mitarbeit danken wir im Namen der DRG, des BDR und des BfS.

Bei etwaigen Unklarheiten stehen wir Ihnen gern telefonisch unter der Rufnummer 030/183332444 oder per E-Mail (dosisct@drg.de) zur Verfügung. 